\title{
Noise Reduction Using a Triple-layer Electrode in Conductive/Capacitive Hybrid Electrocardiogram Measurement
}

\author{
Dairoku Muramatsu ${ }^{1,2 *}$ and Ken Sasaki ${ }^{2}$ \\ ${ }^{1}$ Tokyo University of Science, 2641 Yamazaki, Noda, Chiba 278-8510, Japan \\ ${ }^{2}$ The University of Tokyo, 5-1-5 Kashiwanoha, Kashiwa, Chiba 277-8563, Japan \\ (Received August 18, 2021; accepted September 30, 2021; online published October 11, 2021)
}

Keywords: electrocardiogram, R-R interval, driving, capacitive coupling, noise reduction

The aim of this study was to evaluate a conductive/capacitive hybrid electrocardiogram (ECG) measurement method for monitoring the drowsiness of a driver during car driving. A triple-layer capacitive-coupling electrode comprising driven-shield and sensing electrodes was introduced to reduce the ambient noise, such as the electrostatic induction noise caused by buttock friction. An experiment modeling a car interior showed that the triple-layer electrode successfully reduced the baseline fluctuation and stably measured the ECG R-peaks. The result shows the feasibility of stable $\mathrm{R}-\mathrm{R}$ interval detection during driving using the conductive/ capacitive hybrid ECG measurement system.

\section{Introduction}

The monitoring of various biological signals of drivers when driving, such as the heart rate, blood pressure, and eye movement, has attracted attention as a way to determine the driver's state and to support driving. ${ }^{(1-3)}$ For example, biological signals such as the fluctuation spectrum of the R-R interval (RRI) of an electrocardiogram (ECG) are measured to identify the active and relaxed states of the driver. ${ }^{(4,5)}$ Such measurements allow the detection of whether the driver is drowsy, and driving in a state of drowsiness can be prevented via RRI analysis. ${ }^{(6-8)}$ However, for RRI analysis with practical detection accuracy, it is necessary to measure the RRI stably for at least $1 \mathrm{~min}$. Considering this requirement, the implementation of measurement electrodes, noise in the car, and physiological effects on the driver are serious factors that can affect the detection accuracy. ${ }^{(9,10)}$ Although ECG measurement with a high signal-to-noise ratio (SNR) can be achieved by installing electrodes on both sides of the steering wheel, it is not practical for the driver to hold the steering wheel with both hands for a long time without taking the hands off the wheel. Capacitively coupled electrodes enable the unconstrained measurement of an ECG; however, baseline fluctuations due to environmental and electrostatic induction noise degrade the signal quality. ${ }^{(11-17)}$ In this study, to realize unconstrained and accurate ECG measurement during driving, we explored a measurement method in which conductively and capacitively coupled electrodes are used together (i.e., a conductive/capacitive hybrid ECG measurement

*Corresponding author: e-mail: muramatsu@rs.tus.ac.jp

https://doi.org/10.18494/SAM.2021.3591 
method) (Fig. 1). ${ }^{(18)}$ A triple-layer structure was implemented for the capacitively coupled electrode and evaluated from the viewpoint of noise reduction and RRI detection.

\section{Measurement Setup}

Figure 2 shows the entire measurement setup, modeled as the interior of a car. A human subject sat on a chair and grasped the steering wheel with both hands or the right hand, assuming a driving situation. The ECG of the subject was measured using the conductive/capacitive electrodes, an ECG measurement circuit, and an oscilloscope. Stainless steel electrodes were used as the conductive electrodes in the steering wheel. Three types of capacitively coupled electrode (i.e., a single-layer copper plate, conductive fabric, and triple-layer electrode) were installed on the seat for comparison. The human subject was a Japanese adult male wearing cotton pants. The participation of human subjects was approved by the Research Ethics Committee of Tokyo University of Science, and written informed consent was obtained from the participant prior to the experiment.

\subsection{ECG measurement circuit}

To evaluate the measurement accuracy of the ECG and identify the noise source in an actual environment, we built a measurement circuit in which the cutoff frequency of the filter and the signal gain can be adjusted. Figure 3 shows a block diagram of the ECG measurement circuit. The circuit consists of an instrumentation amplifier with gain $G_{1}$ of 10.7 times, a notch filter with a center frequency $f_{0}=50 \mathrm{~Hz}$, a low-pass filter with cutoff frequency $f_{\mathrm{cl}}=40 \mathrm{~Hz}$, a highpass filter with cutoff frequency $f_{\mathrm{c} 2}=5 \mathrm{~Hz}$, a noninverting amplifier circuit with gain $G_{2}$ of 21 times, and a high-pass filter with cutoff frequency $f_{\mathrm{c} 3}=1.6 \mathrm{~Hz}$. An instrumentation amplifier with a high input impedance of $1 \mathrm{~T} \Omega$ was used to measure the low voltage of the ECG. A $50 \mathrm{~Hz}$ notch filter was used to prevent interference from the commercial power supply. A $5-40 \mathrm{~Hz}$ band-pass filter realized using the low- and high-pass filters was used to detect the R-waves. A 1.6 Hz high-pass filter installed during the final stage was used to remove the offset voltage accumulated in each operational amplifier. Figure 4 shows a schematic of the ECG measurement

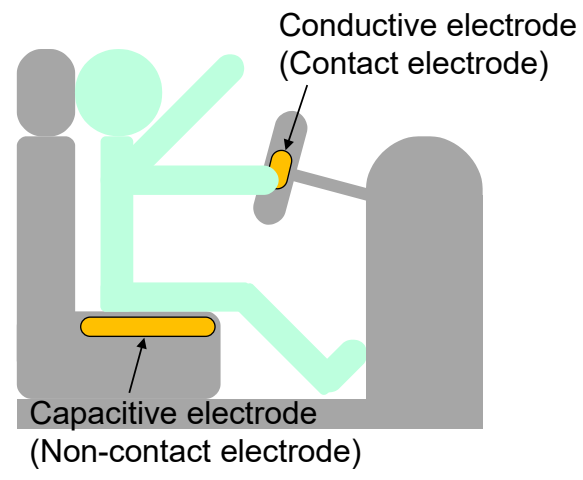

Fig. 1. (Color online) Conductive/capacitive hybrid ECG measurement. 


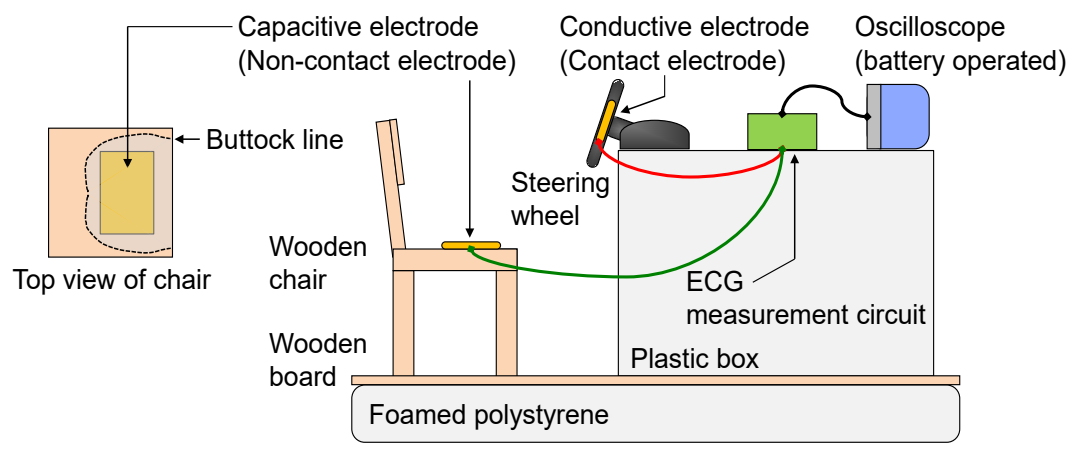

Fig. 2. (Color online) Measurement setup modeling the interior of a car.

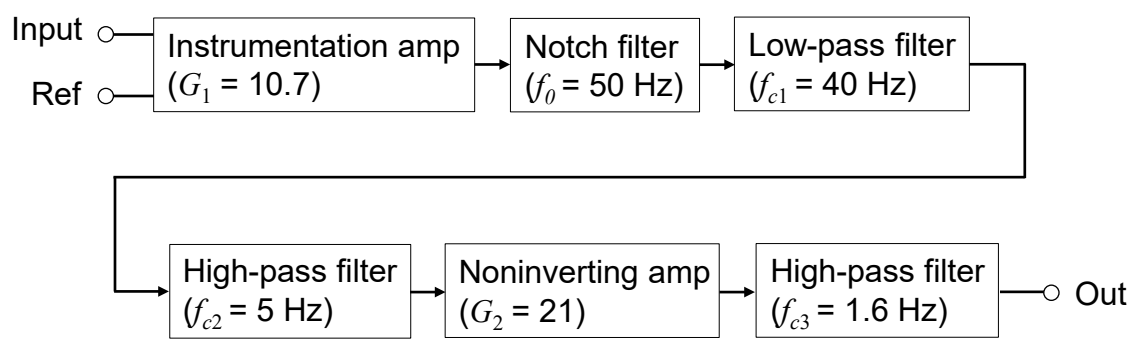

Fig. 3. Block diagram of the ECG measurement circuit.

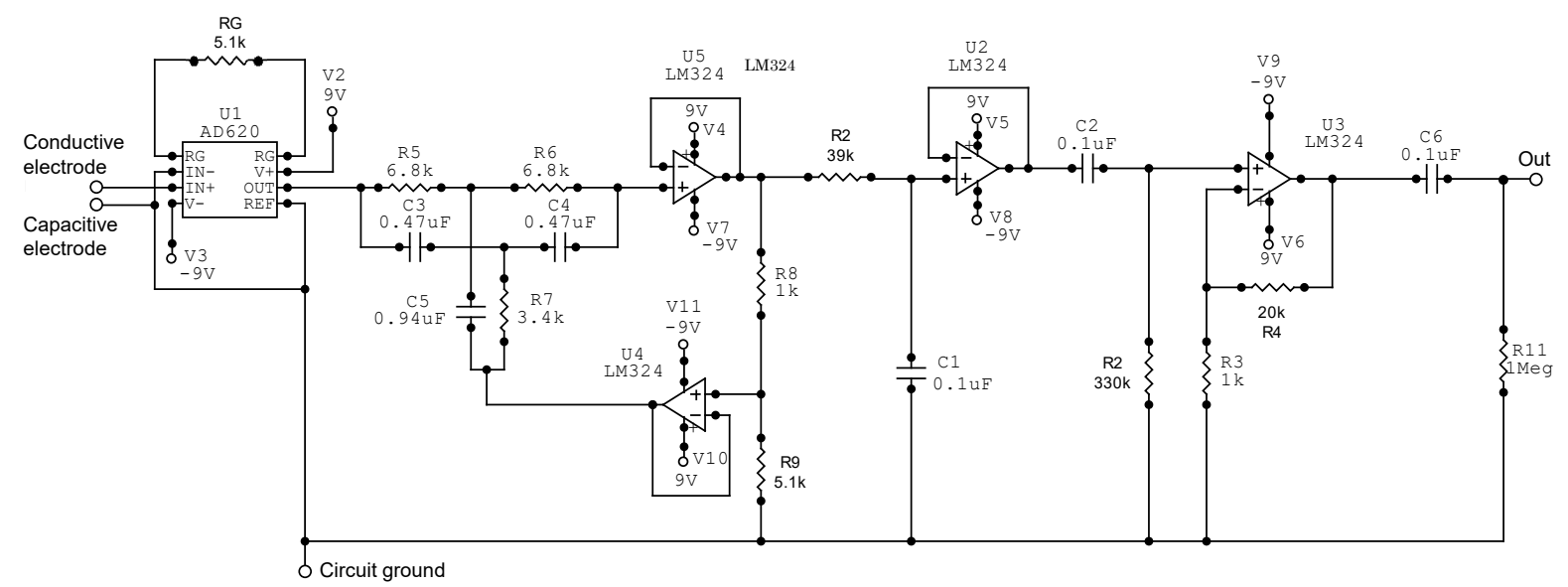

Fig. 4. Schematic of the ECG measurement circuit.

circuit and Fig. 5 shows a photograph of the entire circuit. The instrumentation amplifier was separated from the filter and amplification circuits to improve the SNR against environmental noise that was introduced through the wiring. Furthermore, this separated placement improved the ease of handling of the measurement circuit, such as the connection to the electrodes. The ECG signal processed by the circuit was finally measured using an oscilloscope. 


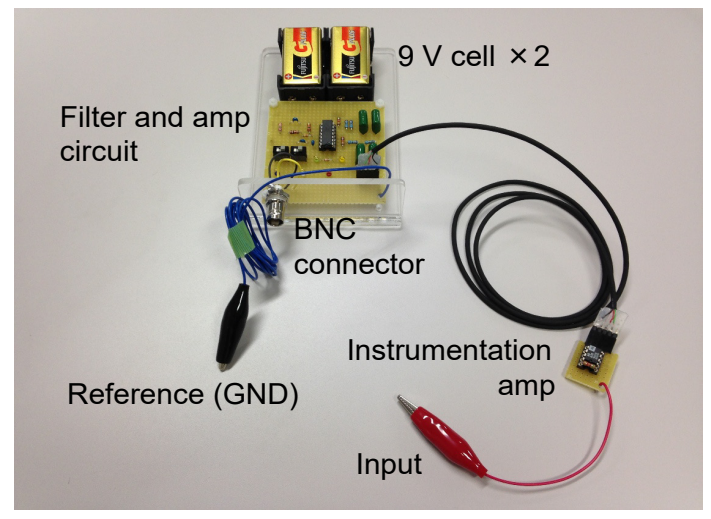

Fig. 5. (Color online) Photograph of the complete ECG measurement circuit.

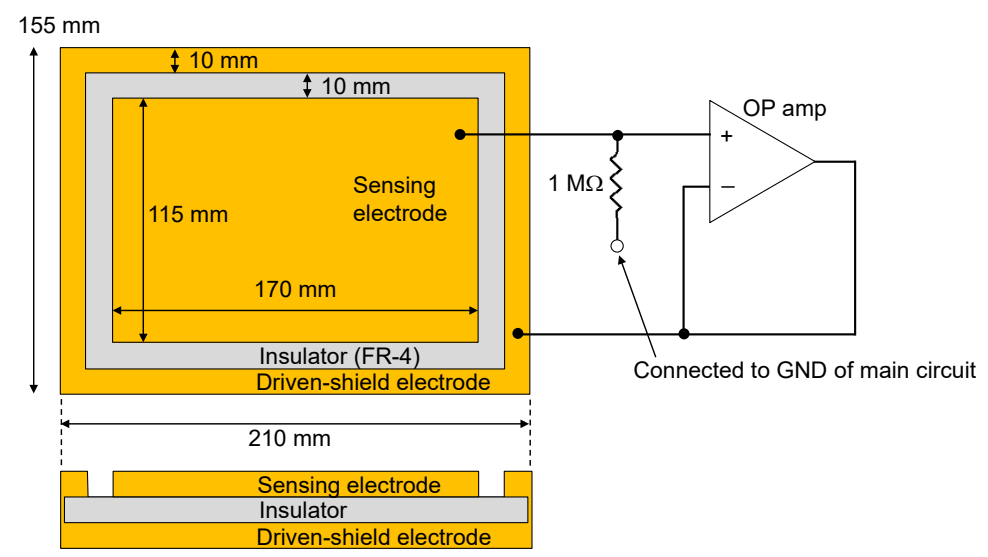

Fig. 6. (Color online) Triple-layer capacitive-coupling electrode.

\subsection{Triple-layer electrode}

Figure 6 shows the triple-layer capacitive-coupling electrode, which comprises driven-shield and sensing electrodes. An FR-4 substrate (thickness $=1.6 \mathrm{~mm}$ ) is inserted between the electrodes as an insulating layer. The driven-shield and sensing electrodes are connected via a voltage follower to maintain them at the same voltage potential. A driven-shield electrode is often implemented to reduce the leakage electric field or current in dielectric measurements and electrostatic sensors. ${ }^{(19)}$ In the ECG measurement, the triple-layer capacitive-coupling electrode is expected to reduce the ambient and electrostatic induction noise caused by buttock friction.

\section{Results and Discussion}

Figure 7 shows four measurement scenarios. Figure 7(a) shows a conductive ECG measurement using the two conductive electrodes placed on the two sides of the steering wheel. Figures 7(b)-7(d) show a conductive/capacitive hybrid ECG measurement using the conductive electrode placed on the right side of the steering wheel and the three types of capacitively coupled electrode on the chair. Figures 8(a)-8(d) show the ECG waveforms measured using the 


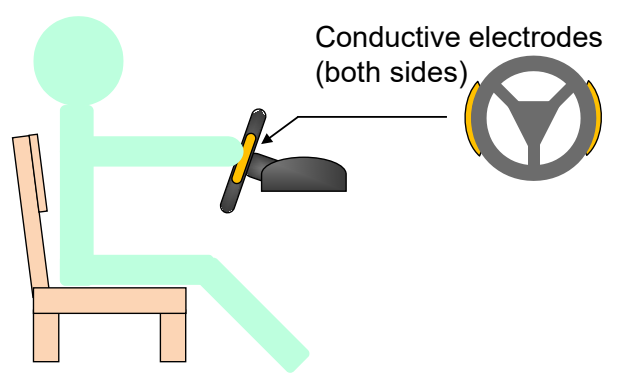

(a)

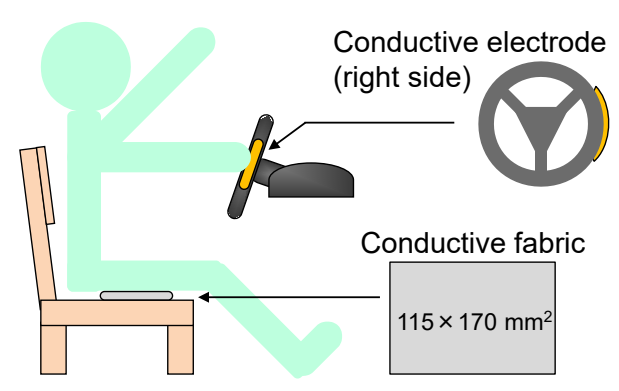

(c)

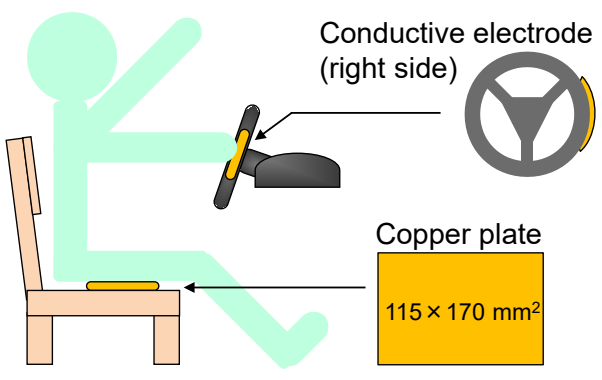

(b)

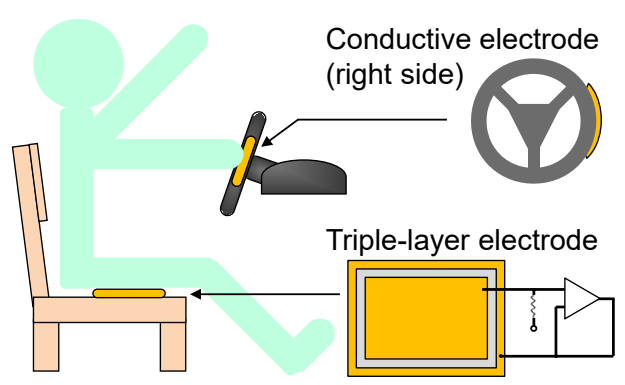

(d)

Fig. 7. (Color online) Measurement scenarios with different combinations of electrodes. (a) Conductive, (b) conductive/capacitive (copper plate), (c) conductive/capacitive (conductive fabric), and (d) conductive/capacitive (triple-layer electrode).

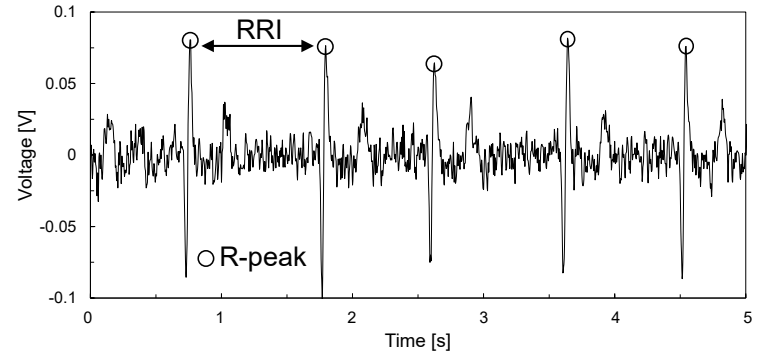

(a)

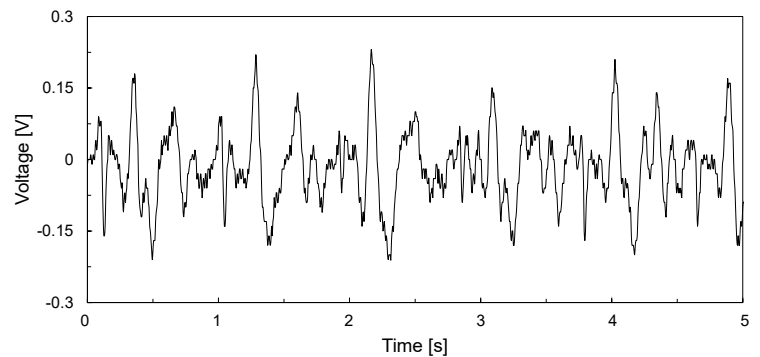

(c)

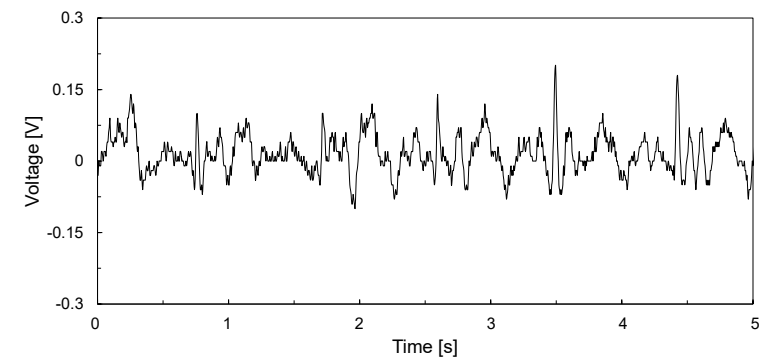

(b)

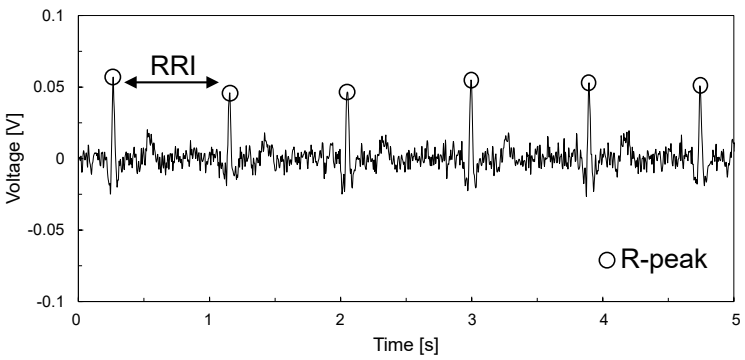

(d)

Fig. 8. (Color online) Measured ECG waveforms. (a) Conductive, (b) conductive/capacitive (copper plate), (c) conductive/capacitive (conductive fabric), and (d) conductive/capacitive (triple-layer electrode). 
setups in Figs. 7(a)-7(d), respectively. As shown in Fig. 8(a), the R-peaks of the ECG can be measured stably when the subject grasps the conductive electrodes on the two sides of the steering wheel. In Fig. 8(b), the ECG waveform is masked by the baseline fluctuation, and the R-peaks are difficult to detect. The baseline fluctuation is mainly caused by electrostatic induction noise due to increased coupling impedance between the body and the capacitive electrode. Meanwhile, the conductive fabric slightly suppresses the baseline fluctuation, as shown in Fig. 8(c). This is because the flexible fabric electrode conforms to the shape of the driver's buttocks, thereby reducing the coupling impedance. However, the stable detection of the R-peak requires advanced signal processing after measurement. Figure 8(d) shows that the triple-layer electrode successfully reduces the baseline fluctuation and measures the R-peaks stably. The suppression of the baseline fluctuation can be attributed to the driven-shield structure surrounding the sensing electrode. The driven-shield and sensing electrodes maintain the same voltage potential when connected using a voltage follower. Thus, the driven-shield electrode absorbs part of the signal that would have flowed into the sensing electrode. Thus, the drivenshield electrode acts as a guard against the electrostatic induction noise caused by buttock friction and other ambient noise. These results show that the R-peak can be detected without signal processing, such as by interpolation and resampling, ${ }^{(20-22)}$ after measurement using a capacitive-coupling electrode with a triple-layer structure.

\section{Conclusions}

In this study, we investigated a conductive/capacitive hybrid ECG measurement system for monitoring the drowsiness of a driver during car driving. Among the examined electrodes, the triple-layer electrode markedly reduced the baseline fluctuation in the ECG waveform to a level low enough to detect RRI without any signal processing. The result shows the feasibility of stable RRI detection during driving using the proposed conductive/capacitive hybrid ECG measurement system. To further improve this system, we will investigate the influence of body movements in the future for practical applications.

\section{Acknowledgments}

This research was partly funded by the Suzuki Foundation and the Futaba Foundation.

\section{References}

1 S. Arimitsu, K. Sasaki, H. Hosaka, M. Itoh, K. Ishida, and A. Ito: IEEE/ASME Trans. Mechatron. 12 (2007) 511. https://doi.org/10.1109/TMECH.2007.905704

2 J. Nishiyama, S. Shinichi, and Y. Hirata: J. Soc. Med. Biol. Eng. 48 (2010) 1. https://doi.org/10.11239/jsmbe.48.1

3 M. Sidikova, R. Martinek, A. Kawala-Sterniuk, M. Ladrova, R. Jaros, L. Danys, and P. Simonik: Sensors 20 (2020) 5699. https://doi.org/10.3390/s20195699

4 R. W. DeBoer, J. M. Karemaker, and J. Strackee: IEEE Trans. Biomed. Eng. 31 (1984) 384. https://doi. org/10.1109/TBME.1984.325351

5 T. Kuusela: Heart Rate Variability (HRV) Signal Analysis (CRC Press, 2012) pp. 9-42.

6 E. Abe, K. Fujiwara, T. Hiraoka, T. Yamakawa, and M. Kano: Signal and Information Processing Association Annual Summit and Conf. (APSIPA, 2014). https://doi.org/10.1109/APSIPA.2014.7041787 
7 S. Muhadi, S. A. Nasution, R. Putranto, and K. Harimurti: Acta Med. Indones. 48 (2016) 48

8 K. Fujiwara, E. Abe, K. Kamata, C. Nakayama, Y. Suzuki, T. Yamakawa, T. Hiraoka, M. Kano, Y. Sumi, F. Masuda, and M. Matsuo: IEEE Trans. Biomed. Eng. 66 (2019) 1769. https://doi.org/10.1109/ TBME.2018.2879346

9 S. Leonhardt and A. Aleksandrowicz: Proc. 5th Int. Summer School Symp. Med. Devices Biosens. (ISSSMDBS, 2008) 183-185. https://doi.org/10.1109/ISSMDBS.2008.4575048

10 B. Chamadiya, S. Heuer, U. G. Hofmann, and M. Wagner: Proc. 4th Eur. Conf.: IFMBE Proc. 22 (2009). https:// doi.org/10.1007/978-3-540-89208-3 291

11 A. Lopez, Jr. and P. C. Richardson: IEEE Trans. Biomed. Eng. 16 (1969) 99. https://doi.org/10.1109/ TBME.2006.889201

12 A. Ueno, Y. Akabane, T. Kato, H. Hoshino, S. Kataoka, and Y. Ishiyama: IEEE Trans. Biomed. Eng. 54 (2007) 759. https://doi.org/10.1109/TBME.2006.889201

13 Y. M. Chi, T. P. Jung, and G. Cauwenberghs: IEEE Rev. Biomed. Eng. 3 (2010) 106. https://doi.org/10.1109/ RBME.2010.2084078

14 B. S. Lin, W. Chou, H. Y. Wang, Y. J. Huang, and J. S. Pan: IEEE J. Transl. Eng. Health Med. 1 (2013) 2700108. https://doi.org/10.1109/JTEHM.2013.2253598

15 H. Tomimori, Y. Ishida, K. Sasaki, Y. Nakano, and S. Sano: Proc. Int. Conf. Machine Automation (ICMA, 2008).

16 M. Takano and A. Ueno: IEEE J. Biomed. Health Inform. 23 (2019) 618. https://doi.org/10.1109/ JBHI.2018.2825020

17 T.-W. Wang, H. Zhang, and S.-F. Lin: IEEE Sens. J. 20 (2020) 9265. https://doi.org/10.1109/JSEN.2020.2986723

18 Y. Kondo, K. Sasaki, Y. Nakano, S. Sano, and H. Tomimori: TENCON 2010-2010 IEEE Region 10 Conf. (IEEE, 2010) 1738-1741. https://doi.org/10.1109/TENCON.2010.5686134

19 X. Hu and W. Yang: Sens. Rev. 30 (2010) 24. https://doi.org/10.1108/02602281011010772

20 G. D. Clifford and L. Tarassenko: IEEE Trans. Biomed. Eng. 52 (2005) 630. https://doi.org/10.1109/ TBME.2005.844028

21 K. Kamata, K. Fujiwara, T. Yamakawa, and M. Kano: 38th Annu. Int. Conf. IEEE Engineering in Medicine and Biology Society (EMBC) (IEEE, 2016) 2386-2389. https://doi.org/10.1109/EMBC.2016.7591210

22 R. Aoki, K. Eguchi, S. Shimauchi, K. Yoshida, and T. Yamada: 40th Annu. Int. Conf. IEEE Engineering in Medicine and Biology Society (EMBC) (IEEE, 2018) 5693-5696. https://doi.org/10.1109/EMBC.2018.8513449 